\title{
Volume 35 Edição 3 - Set/Out 2013
}

\section{Peritonites em pacientes em diálise peritoneal: análise de um centro brasileiro segundo as recomendações da Sociedade Brasileira de Diálise Peritoneal}

Peritonitis in patients on peritoneal dialysis: analysis of a single Brazilian center based on the International Society for Peritoneal Dialysis

Ana Elizabeth Figueiredo, Carlos Eduardo Poli-de-Figueiredo, Franciele Meneghetti, Gonzalo Aejandro Pacheco Lise, Caroline Costa Detofoli, Luisa Bicca da Silva

O título foi publicado como Peritonites em pacientes em diálise peritoneal: análise de um centro brasileiro segundo as recomendações da Sociedade Brasileira de Diálise Peritoneal e o correto é Peritonites em pacientes em diálise peritoneal: análise de um único centro brasileiro segundo a Sociedade Internacional de Diálise Peritoneal. 